\title{
Conditions de logement et état de santé des aînés d'après l'Enquête sur la santé dans les collectivités canadiennes de 2018
}

\author{
Sebastian A. Srugo, B.H.S.; Ying Jiang, M.D., M. Sc.; Margaret de Groh, Ph. D.
}

Diffuser cet article sur Twitter

\begin{abstract}
Résumé
À l'heure actuelle, au Canada, un aîné sur trois répond aux critères d'un vieillissement réussi, soit une faible probabilité de maladie et d'incapacité, des aptitudes cognitives et physiques élevées et une participation active à la vie. Les caractéristiques sociodémographiques des personnes vivant seules permettent de cibler les aînés à risque élevé, étant donné l'association avec un soutien et des interactions sociales souvent plus faibles, ce qui rend cette population plus susceptible de souffrir de problèmes de santé à un âge avancé. Il existe pourtant peu de données sur les conditions de logement des aînés canadiens. Dans cette analyse, nous présentons les caractéristiques sociodémographiques et les mesures de la santé et du bien-être social des personnes âgées en fonction de leurs conditions de logement. Ces données devraient servir à identifier et à soutenir les aînés vulnérables et à accroître la prévalence d'un vieillissement en santé chez les Canadiens.
\end{abstract}

Mots-clés : conditions de logement, aînés, vieillissement en santé

\section{Introduction}

En raison de l'augmentation de l'espérance de vie $^{1}$ et de la baisse des taux de fécondité 2 la proportion d'aînés au Canada augmente plus rapidement que jamais. En 2011, cette tendance a commencé à s'accélérer lorsque les premiers baby-boomers ont fêté leurs 65 ans. Les aînés sont maintenant plus de 6 millions (1 personne sur 6) au Canada ${ }^{3}$ et, pour la première fois, sont plus nombreux que les enfants de 0 à 14 ans $^{4}$. De plus, les tendances actuelles laissent penser que ce groupe d'âge continuera à augmenter : la proportion d'aînés devrait atteindre 1 sur 5 d'ici 2024 et 1 sur 4 d'ici $2055^{3}$. Il sera de plus en plus important de mettre en œuvre des politiques et des programmes visant à promouvoir la santé des personnes âgées, car seulement environ 1 aîné sur 3 répond actuellement aux critères d'un vieillissement réussi ${ }^{5}$ définis par Rowe et $\mathrm{Kahn}^{6}$ : une faible probabilité de maladie et d'incapacité, des aptitudes cognitives et physiques élevées et une participation active à la vie.
Des études internationales récentes ont montré que les conditions de logement des aînés sont un facteur déterminant d'un vieillissement en santé, dans la mesure où elles permettent de prédire le soutien social et les interactions. On a ainsi montré que les aînés vivant en couple étaient plus susceptibles d'avoir des taux d'incidence plus faibles de démence ${ }^{7}$, une meilleure santé mentale et moins de limitations à leur participation à toutes les dimensions de la vie (vie sociale, travaux ménagers et activités de loisirs) dues à la multimorbidité ${ }^{8}$, que ceux qui vivaient avec des membres de leur famille présentaient des taux moindres de maladies chroniques et aiguës ${ }^{9}$, et que ceux qui vivaient avec d'autres personnes faisaient état d'une meilleure santé mentale et de davantage de soutien social et de la pratique de davantage d'activités physiques que ceux vivant seuls ${ }^{10}$. Toutefois, seules quelques études ont évalué les conditions de logement des aînés canadiens ${ }^{11-14}$ et aucune n'a tenté de cibler les souspopulations d'aînés plus susceptibles de

\section{Points saillants}

- Une compréhension des conditions de logement pourrait aider les gens qui développent les programmes d'intervention à mieux cibler les aînés courant un risque plus élevé de problèmes de santé à un âge avancé.

- D'après nos résultats, les femmes, les personnes plus âgées, à faible revenu, divorcées ou séparées, vivant en milieu urbain, locataires et moins scolarisées étaient plus susceptibles de vivre seules.

- Les aînés vivant seuls étaient également plus susceptibles de faire état d'une perception défavorable de leur santé et de leur bien-être social.

- Ces résultats peuvent servir à mieux cibler les politiques et les programmes visant à améliorer l'état de santé des aînés.

vivre seuls, condition qui les rend plus à risque de souffrir de problèmes de santé à un âge avancé. De plus, la moitié de ces études portaient uniquement sur les aînés canadiens d'origine asiatique ${ }^{13,14}$. Des données récentes et exhaustives sur ce sujet sont nécessaires pour cerner et combler les lacunes dans la promotion d'un vieillissement en santé chez les aînés.

L'objectif de cette brève analyse était d'examiner les conditions de logement des aînés canadiens dans l'Enquête sur la santé dans les collectivités canadiennes (ESCC) la plus réecente (2018) en fonction de critères sociodémographiques, de santé

Rattachement des auteurs :

Agence de la santé publique du Canada, Ottawa (Ontario) Canada

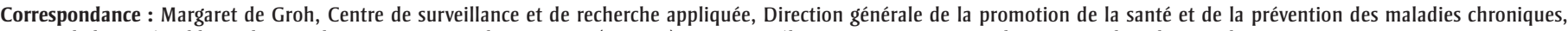
Agence de la santé publique du Canada, 785, avenue Carling, Ottawa (Ontario) K1S 5H4, tél. : 613-614-2045, courriel : Margaret.degroh@canada.ca 
et de bien-être social, stratifiés selon le sexe.

\section{Méthodologie}

L'ESCC est une enquête transversale annuelle qui recueille des données représentatives sur l'état de santé et les déterminants de la santé de la population canadienne ne résidant pas en établissement, et ce, dans toutes les provinces ${ }^{15}$. Les habitants des territoires ont été exclus de la composante annuelle en raison de la petite taille des échantillons et de leur absence de représentativité ${ }^{15}$. Nous avons utilisé les données du cycle de l'ESCC de 2018 correspondant aux personnes de 65 ans et plus vivant dans en ménage privé et ayant répondu à la question sur les conditions de logement. Nous avons utilisé, pour cette analyse, l'information sur les conditions de logement, les caractéristiques sociodémographiques (groupe d'âge, origine ethnique, quintile de revenu du ménage au niveau de la région sanitaire, état matrimonial, région de résidence, type de milieu [urbain ou rural], propriété de la résidence, niveau de scolarisation du répondant et taille du ménage) et le bienêtre autodéclaré (santé perçue, santé mentale perçue, satisfaction dans la vie et sens d'appartenance à la collectivité). Le ratio de revenu du ménage mesure le revenu du ménage d'un répondant par rapport à celui des autres résidents de sa région sanitaire, ajusté pour la taille du ménage et de la collectivité ${ }^{15}$. Un milieu urbain (centre de population) est défini comme une zone comptant au moins 1000 habitants et ayant une densité minimale de 400 personnes au kilomètre carré. Toutes les autres zones sont considérées comme rurales $^{15}$. Nous avons stratifié les statistiques descriptives selon le sexe et les avons pondérées avec des méthodes d'autoamorçage (bootstrap) afin de produire des données représentatives de la population d'aînés canadiens habitant dans les provinces. Il importe de noter qu'en 2016, l'ESCC contenait une question demandant aux répondants s'ils étaient un homme ou une femme. Nous reconnaissons que le genre d'une personne influence ses perceptions et ses comportements, et cela devrait être pris en compte lors de l'interprétation de nos résultats. Les données sont présentées en pourcentage avec des intervalles de confiance (IC) à $95 \%$ respectant la méthode d'autoamorçage et ont été comparées avec le test Rao-Scott $\chi^{2}$. Toutes les analyses ont été effectuées à l'aide de la version 5.1 du logiciel SAS
TABLEAU 1

Conditions de logement de 8261 femmes aînées, ESCC 2018, échantillon pondéré et stratifié selon diverses caractéristiques

\begin{tabular}{|c|c|c|c|c|}
\hline Caractéristiques & $\begin{array}{l}\text { Femmes vivant } \\
\text { seules }(n=4 \text { 313) }\end{array}$ & $\begin{array}{l}\text { Femmes vivant en } \\
\text { couple }^{a}(n=3214)\end{array}$ & Autres $^{b}(n=734)$ & $\begin{array}{l}\text { Valeur } \\
\text { de } p\end{array}$ \\
\hline Effectif pondéré (\%) & $1170194(35,7)$ & $1604305(48,9)$ & $507312(15,5)$ & \\
\hline Groupe d'âge & & & & $<0,001$ \\
\hline 65 à 74 ans & $28,4(26,3$ à 30,4$)$ & 57,1 (54,8 à 59,5) & $14,5$ (12,3 à 16,7$)$ & \\
\hline 75 à 84 ans & 42,6 (39,5 à 45,7) & 43,0 (39,7 à 46,3) & 14,5 (11,5 à 17,4) & \\
\hline 85 ans ou plus & $58,4(53,1$ à 63,6) & 18,3 (13,7 à 22,9) & 23,3 (18,3 à 28,4) & \\
\hline Origine ethnique & & & & $<0,001$ \\
\hline Blanc & $36,8(35,0$ à 38,6$)$ & 50,9 (49,1 à 52,7) & $12,3(10,8$ à 13,9$)$ & \\
\hline Autochtone & $34,1(25,6$ à 42,6) & 51,3 (41,0 à 61,7) & 14,6 (7,6 à 21,6) & \\
\hline Asiatique $^{c}$ & $16,0(10,6$ à 21,4$)$ & 38,7 (30,1 à 47,2) & 45,3 (35,9 à 54,8) & \\
\hline Autres $^{d}$ & $38,6(28,2$ à 49,0) & 34,3 (24,3 à 44,3) & 27,1 (16,3 à 37,8) & \\
\hline \multicolumn{4}{|c|}{ Ratio de revenu du ménage au niveau de la région sanitaire (quintiles)e } & $<0,001$ \\
\hline 1 & $54,2(50,9$ à 57,5$)$ & $32,1(29,0$ à 35,2$)$ & $13,7(11,0$ à 16,4$)$ & \\
\hline 2 & $35,7(32,5$ à 38,9) & $48,0(44,6$ à 51,4) & $16,3(13,1$ à 19,4$)$ & \\
\hline 3 & $29,1(25,8$ à 32,5$)$ & $55,9(51,9$ à 60,0$)$ & $14,9(10,9$ à 18,9$)$ & \\
\hline 4 & $22,6(19,3$ à 26,0$)$ & $58,9(53,7$ à 64,1$)$ & $18,5(13,1$ à 23,9$)$ & \\
\hline 5 & $20,6(16,8$ à 24,5$)$ & $64,9(59,8$ à 70,1$)$ & $14,4(10,6$ à 18,3$)$ & \\
\hline État matrimonial & & & & S.o. ${ }^{f}$ \\
\hline Mariée ou conjointe de fait & $1,6(1,2$ à 1,9$)$ & $92,6(91,2$ à 94,0$)$ & $5,8(4,4$ à 7,2$)$ & \\
\hline Veuve & $73,7(70,3$ à 77,2) & 0 & $26,3(22,8$ à 29,7$)$ & \\
\hline Divorcée ou séparée & $76,9(71,1$ à 82,8$)$ & 0 & 23,1 (17,2 à 28,9) & \\
\hline Célibataire & $66,9(58,2$ à 75,6$)$ & 0 & $33,1(24,4$ à 41,8) & \\
\hline Région de résidence 8 & & & & 0,2 \\
\hline Atlantique & $34,4(31,0$ à 37,9$)$ & 53,3 (49,6 à 57,0) & 12,3 (9,5 à 15,1) & \\
\hline Centre & $35,8(33,5$ à 38,0$)$ & $48,0(45,5$ à 50,5$)$ & $16,2(14,0$ à 18,5$)$ & \\
\hline Prairies & 37,6 (33,9 à 41,3) & 49,5 (45,7 à 53,2) & $12,9$ (9,0 à 16,8$)$ & \\
\hline Ouest & $33,8(30,3$ à 37,3$)$ & 50,0 (45,5 à 54,5) & $16,2(12,5$ à 19,9$)$ & \\
\hline Milieu $^{\mathrm{h}}$ & & & & $<0,001$ \\
\hline Urbain (centre de population) & $37,7(35,7$ à 39,7) & 46,2 (44,0 à 48,4) & $16,1(14,1$ à 18,1$)$ & \\
\hline Rural & $26,7(24,5$ à 28,8) & 60,6 (57,8 à 63,3) & $12,7(10,4$ à 15,0$)$ & \\
\hline Propriété de la résidence & & & & $<0,001$ \\
\hline Propriétaire & $26,9(25,3$ à 28,6$)$ & 56,7 (54,7 à 58,7) & $16,4(14,5$ à 18,2$)$ & \\
\hline Locataire & $62,4(58,4$ à 66,4) & 25,0 (21,7 à 28,3) & 12,6 (9,1 à 16,2) & \\
\hline Scolarité & & & & $<0,001$ \\
\hline Sans DES & $41,0(37,5$ à 44,4$)$ & $38,6(35,1$ à 42,1) & 20,4 (16,9 à 23,9) & \\
\hline DES & $35,3(32,0$ à 38,5$)$ & $52,7(49,0$ à 56,4) & 12,0 (9,4 à 14,6) & \\
\hline Diplôme d'études postsecondaires & 33,3 (30,9 à 35,7) & $52,5(49,7$ à 55,2) & $14,2(11,5$ à 16,9$)$ & \\
\hline
\end{tabular}

Abréviations : DES, diplôme d'études secondaires; ESCC, Enquête sur la santé dans les collectivités canadiennes; IC, intervalle de confiance.

Remarques : Sauf indication contraire, les données sont exprimées en pourcentage pour la rangée (IC à 95 \%).

a Avec ou sans enfants.

${ }^{\mathrm{b}}$ Vivant avec des enfants ou d'autres personnes, apparentées ou non.

' Asiatiques du Sud, de l'Ouest et du Sud-Est, Chinois, Coréens, Japonais et Philippins.

d Noirs, Latino-Américains, Arabes et personnes d'autres origines ethniques ou appartenant à plusieurs groupes ethniques.

e Répartition des participants dans chaque région sanitaire selon le ratio ajusté du revenu total du ménage par rapport au seuil de faible revenu correspondant à la taille du ménage et de la collectivité.

${ }^{\mathrm{f}}$ Aucun test ne peut être calculé pour le tableau puisqu'au moins une cellule a une fréquence de zéro.

$\&$ L'Atlantique comprend I'Île-du-Prince-Édouard, le Nouveau-Brunswick, la Nouvelle-Écosse et Terre-Neuve-et-Labrador, le Centre comprend le Québec et l'Ontario, les Prairies comprennent l'Alberta, le Manitoba et la Saskatchewan et l'Ouest comprend la Colombie-Britannique.

${ }^{\mathrm{h}}$ Un centre de population est défini comme une zone d'au moins 1000 habitants et d'une densité minimale de 400 personnes au kilomètre carré; toutes les autres zones sont considérées comme des zones rurales. 
Enterprise Guide (SAS Institute Inc., Cary, Caroline du Nord, États-Unis).

\section{Résultats}

Au total, 8261 aînées et 6532 aînés ayant répondu à l'ESCC de 2018 ont été inclus dans nos analyses. Les données sur les conditions de logement des femmes (tableau 1) et celles des hommes (tableau 2) ont été pondérées et stratifiées selon les caractéristiques choisies. Les femmes étaient presque deux fois plus susceptibles de vivre seules $(35,7 \%$ contre $19,1 \%)$ et 1,5 fois moins susceptibles d'habiter en couple $(48,9 \%$ contre $71,3 \%)$ que les hommes. Pour les deux sexes, la probabilité de vivre seul augmentait pour les participants plus âgés, à faible revenu, divorcés ou séparés, vivant en milieu urbain, locataires et moins scolarisés. Les caractéristiques opposées étaient associées à une vie en couple (répondant plus jeune, à revenu plus élevé, marié ou en union libre, résidant en milieu rural, propriétaire et plus scolarisé). Les Asiatiques étaient plus susceptibles de vivre avec des enfants ou d'autres personnes (apparentées ou non) et moins susceptibles de vivre seuls (pour les deux sexes). Pour les aînés des deux sexes, les conditions de logement ne différaient pas selon la région. Quelques différences entre les sexes ont toutefois pu être établies. Chez les femmes, les participantes blanches étaient plus susceptibles de vivre en couple que les Asiatiques et les personnes d'origines ethniques " autres » (p. ex. noires, latino-américaines et arabes), alors qu'aucune différence en fonction de l'origine ethnique n'a été observée chez les hommes vivant en couple. Les femmes locataires étaient beaucoup plus susceptibles de vivre seules qu'en couple $(62,4 \%$ contre $25 \%$ ), mais aucune tendance semblable n'a été observée chez les hommes locataires (45,5\% contre 47,9\%). Enfin, la prévalence de la vie de couple augmentait avec le niveau de scolarité chez les deux sexes, quoique le plus grand écart ait été observé entre les femmes n'ayant pas terminé leurs études secondaires et celles ayant obtenu leur diplôme d'études secondaires (femmes : 38,6\% contre 52,7 \%; hommes : $64,9 \%$ contre $74,1 \%$ ).

Les mesures de la santé et du bien-être social perçus ont également été stratifiées selon le sexe et les conditions de logement (tableau 3). Pour l'ensemble des quatre mesures, les aînés vivant en couple étaient moins susceptibles de déclarer une moins bonne santé ou un bien-être social plus
TABLEAU 2

Conditions de logement de 6532 hommes aînés, ESCC 2018, échantillon pondéré et stratifié selon diverses caractéristiques

\begin{tabular}{|c|c|c|c|c|}
\hline & $\begin{array}{l}\text { Hommes vivant } \\
\text { seuls }(n=2 \text { 105) }\end{array}$ & $\begin{array}{l}\text { Hommes vivant en } \\
\text { couple }^{\mathrm{a}}(n=4 \text { 065) }\end{array}$ & Autres $^{b}(n=362)$ & $\begin{array}{l}\text { Valeur } \\
\text { de } p\end{array}$ \\
\hline Nbre pondéré (\%) & $540770(19,1)$ & $2022160(71,3)$ & $273833(9,7)$ & \\
\hline Groupe d'âge & & & & $<0,001$ \\
\hline 65 à 74 ans & $17,5$ (15,9 à 19,0$)$ & 73,4 (71,2 à 75,6) & 9,1 (7,3 à 10,9) & \\
\hline 75 à 84 ans & $20,0(17,6$ à 22,4$)$ & 71,1 (68,1 à 74,1) & $8,9(6,6$ à 11,1$)$ & \\
\hline 85 ans ou plus & 28,3 (23,3 à 33,4) & 54,5 (48,0 à 61,0) & $17,1(10,9$ à 23,4$)$ & \\
\hline Origine ethnique & & & & $<0,001$ \\
\hline Blanc & $20,6(19,1$ à 22,0$)$ & 72,0 (70,3 à 73,8) & 7,4 (6,1 à 8,7) & \\
\hline Autochtone & $20,0(11,4$ à 28,6$)$ & 63,8 (52,8 à 74,8) & $16,2(7,0$ à 25,4$)$ & \\
\hline Asiatique $^{c}$ & 8,5 (3,8 à 13,2) & 67,8 (58,7 à 76,9) & $23,7(15,2$ à 32,2$)$ & \\
\hline Autres $^{d}$ & $13,7$ (7,4 à 20,1$)$ & $69,6(60,0$ à 79,2$)$ & 16,7 (8,6 à 24,8) & \\
\hline \multicolumn{4}{|c|}{ Ratio du revenu du ménagé au niveau de la région sanitaire (quintiles)e } & $<0,001$ \\
\hline 1 & $30,7(27,4$ à 34,0$)$ & 57,8 (54,0 à 61,7) & $11,5(8,6$ à 14,3$)$ & \\
\hline 2 & $18,0(15,5$ à 20,4$)$ & 71,0 (67,5 à 74,6) & 11,0 (7,8 à 14,2) & \\
\hline 3 & $15,8(13,6$ à 18,0$)$ & 74,4 (70,8 à 77,9) & $9,9(6,5$ à 13,2$)$ & \\
\hline 4 & $12,5(10,0$ à 15,0$)$ & $80,9(77,6$ à 84,3) & $6,6(4,1$ à 9,1$)$ & \\
\hline 5 & $14,6(11,6$ à 17,5$)$ & 78,2 (74,2 à 82,1) & $7,3(4,4$ à 10,2$)$ & \\
\hline État matrimonial & & & & S.o. ${ }^{f}$ \\
\hline Marié ou conjoint de fait & $1,3(0,9$ à 1,6$)$ & 92,1 (90,7 à 93,5) & $6,6(5,2$ à 8,0$)$ & \\
\hline Veuf & $78,4(73,7$ à 83,2$)$ & 0 & $21,6(16,8$ à 26,3$)$ & \\
\hline Divorcé ou séparé & $83,0(77,3$ à 88,6$)$ & 0 & $17,0(11,4$ à 22,7$)$ & \\
\hline Célibataire & $78,0(69,7$ à 86,2$)$ & 0 & $22,0(13,8$ à 30,3$)$ & \\
\hline Région de résidence 8 & & & & 0,07 \\
\hline Atlantique & $18,5$ (15,5 à 21,6$)$ & 75,1 (71,8 à 78,5) & 6,3 (4,2 à 8,4) & \\
\hline Centre & $19,6(17,9$ à 21,3$)$ & $71,2(68,9$ à 73,5) & $9,2(7,3$ à 11,1$)$ & \\
\hline Prairies & $16,1(13,6$ à 18,5$)$ & 73,2 (69,5 à 76,9) & $10,7(7,3$ à 14,0$)$ & \\
\hline Ouest & $20,0(16,7$ à 23,2$)$ & 67,8 (63,3 à 72,3) & $12,2(8,3$ à 16,2$)$ & \\
\hline Milieuh $^{\mathrm{h}}$ & & & & 0,02 \\
\hline Urbain (centre de population) & $19,6(18,0$ à 21,2$)$ & 70,2 (68,1 à 72,2) & 10,2 (8,5 à 11,9) & \\
\hline Rural & $17,2(15,3$ à 19,0$)$ & 75,1 (72,6 à 77,6) & 7,7 (5,7 à 9,8) & \\
\hline Propriété de la résidence & & & & $<0,001$ \\
\hline Propriétaire & $13,6(12,4$ à 14,7$)$ & 76,3 (74,6 à 78,1) & $10,1(8,5$ à 11,7$)$ & \\
\hline Locataire & $45,5(40,8$ à 50,1$)$ & 47,9 (42,9 à 52,9) & 6,7 (4,0 à 9,3) & \\
\hline Scolarité & & & & $<0,001$ \\
\hline Sans DES & $23,2(20,5$ à 25,9$)$ & 64,9 (61,2 à 68,5) & $12,0$ (8,8 à 15,1$)$ & \\
\hline DES & $22,8(19,2$ à 26,3$)$ & 69,2 (65,2 à 73,1) & $8,1(5,3$ à 10,8$)$ & \\
\hline Diplôme d'études postsecondaires & $17,0(15,3$ à 18,6$)$ & 74,1 (71,8 à 76,3) & $9,0$ (7,1 à 10,8$)$ & \\
\hline
\end{tabular}

Abréviations : DES, diplôme d'études secondaires; ESCC, Enquête sur la santé dans les collectivités canadiennes; IC, intervalle de confiance.

Remarques : Sauf indication contraire, les données sont exprimées en pourcentagé pour la rangée (IC à $95 \%$ ).

avec ou sans enfants.

${ }^{\mathrm{b}}$ Vivant avec des enfants ou d'autres personnes, apparentées ou non.

'Asiatiques du Sud, de l'Ouest et du Sud-Est, Chinois, Coréens, Japonais et Philippins.

¿ Noirs, Latino-Américains, Arabes et personnes d'autres origines ethniques ou appartenant à plusieurs groupes ethniques.

e Répartition des participants dans chaque région sanitaire selon le ratio ajusté du revenu total du ménage par rapport au seuil de faible revenu correspondant à la taille du ménage et de la collectivité.

${ }^{\text {† }}$ Aucun test ne peut être calculé pour le tableau puisqu'au moins une cellule a une fréquence de zéro.

${ }^{8}$ L'Atlantique comprend l'île-du-Prince-Édouard, le Nouveau-Brunswick, la Nouvelle-Écosse et Terre-Neuve-et-Labrador, le Centre comprend le Québec et l'Ontario, les Prairies comprennent l'Alberta, le Manitoba et la Saskatchewan et l'Ouest comprend la Colombie-Britannique.

${ }^{\text {h }}$ Un centre de population est défini comme une zone d'au moins 1000 habitants et d'une densité minimale de 400 personnes au kilomètre carré; toutes les autres zones sont considérées comme des zones rurales. 
TABLEAU 3

Mesures de la santé et du bien-être social perçus chez les participants à l'ESCC de 2018, échantillon pondéré et stratifié selon le sexe et les conditions de logement

\begin{tabular}{|c|c|c|c|c|c|c|c|c|}
\hline & \multicolumn{2}{|c|}{ Santé globale perçue } & \multicolumn{2}{|c|}{ Santé mentale perçue } & \multicolumn{2}{|c|}{ Satisfaction à l'égard de la vie } & \multicolumn{2}{|c|}{$\begin{array}{c}\text { Sentiment d'appartenance } \\
\text { à la collectivité }\end{array}$} \\
\hline & $\begin{array}{l}\text { Moins que } \\
\text { très bonne }\end{array}$ & $\begin{array}{c}\text { Très bonne ou } \\
\text { excellente }\end{array}$ & $\begin{array}{l}\text { Moins que } \\
\text { très bonne }\end{array}$ & $\begin{array}{l}\text { Très bonne ou } \\
\text { excellente }\end{array}$ & $\begin{array}{l}\text { Moins que } \\
\text { satisfait }\end{array}$ & $\begin{array}{l}\text { Satisfait ou } \\
\text { très satisfait }\end{array}$ & $\begin{array}{l}\text { Assez ou très } \\
\text { faible }\end{array}$ & $\begin{array}{l}\text { Assez ou très } \\
\text { fort }\end{array}$ \\
\hline Femmes & \multicolumn{2}{|c|}{$p<0,001$} & \multicolumn{2}{|c|}{$p=0,001$} & \multicolumn{2}{|c|}{$p<0,001$} & \multicolumn{2}{|c|}{$p<0,001$} \\
\hline \multicolumn{9}{|c|}{ Conditions de logement } \\
\hline Vivant seules & $\begin{array}{c}53,4 \\
(51,1 \text { à } 55,7)\end{array}$ & $\begin{array}{c}46,6 \\
(44,3 \text { à 48,9) }\end{array}$ & $\begin{array}{c}31,2 \\
(28,9 \text { à } 33,6)\end{array}$ & $\begin{array}{c}68,8 \\
(66,4 \text { à } 71,1)\end{array}$ & $\begin{array}{c}11,9 \\
(10,3 \text { à } 13,6)\end{array}$ & $\begin{array}{c}88,1 \\
(86,4 \text { à } 89,7)\end{array}$ & $\begin{array}{c}24,8 \\
(22,7 \text { à } 26,9)\end{array}$ & $\begin{array}{c}75,2 \\
(73,1 \text { à } 77,3)\end{array}$ \\
\hline Vivant en couple ${ }^{\mathrm{a}}$ & $\begin{array}{c}46,9 \\
(44,3 \text { à 49,6) }\end{array}$ & $\begin{array}{c}53,1 \\
(50,4 \text { à } 55,7)\end{array}$ & $\begin{array}{c}25,9 \\
(23,6 \text { à } 28,1)\end{array}$ & $\begin{array}{c}74,1 \\
(71,9 \text { à } 76,4)\end{array}$ & $\begin{array}{c}7,1 \\
(5,7 \text { à } 8,4)\end{array}$ & $\begin{array}{c}92,9 \\
(91,6 \text { à } 94,3)\end{array}$ & $\begin{array}{c}21,7 \\
(19,4 \text { à } 24,1)\end{array}$ & $\begin{array}{c}78,3 \\
(75,9 \text { à } 80,6)\end{array}$ \\
\hline Autres $^{b}$ & $\begin{array}{c}61,7 \\
(55,2 \text { à } 68,3)\end{array}$ & $\begin{array}{c}38,3 \\
(31,7 \text { à 44,8) }\end{array}$ & $\begin{array}{c}35,2 \\
(28,8 \text { à } 41,7)\end{array}$ & $\begin{array}{c}64,8 \\
(58,3 \text { à } 71,2)\end{array}$ & $\begin{array}{c}11,7 \\
(8,1 \text { à } 15,2)\end{array}$ & $\begin{array}{c}88,3 \\
(84,8 \text { à } 91,9)\end{array}$ & $\begin{array}{c}35,7 \\
(29,1 \text { à } 42,2)\end{array}$ & $\begin{array}{c}64,3 \\
(57,8 \text { à } 70,8)\end{array}$ \\
\hline Hommes & \multicolumn{2}{|c|}{$p=0,01$} & \multicolumn{2}{|c|}{$p=0,007$} & \multicolumn{2}{|c|}{$p<0,001$} & \multicolumn{2}{|c|}{$p=0,003$} \\
\hline \multicolumn{9}{|c|}{ Conditions de logement } \\
\hline Vivant seuls & $\begin{array}{c}56,5 \\
(53,2 \text { à } 59,8)\end{array}$ & $\begin{array}{c}43,5 \\
(40,2 \text { à } 46,8)\end{array}$ & $\begin{array}{c}31,7 \\
(28,9 \text { à 34,6) }\end{array}$ & $\begin{array}{c}68,3 \\
(65,4 \text { à } 71,1)\end{array}$ & $\begin{array}{c}13,0 \\
(11,1 \text { à } 15,0)\end{array}$ & $\begin{array}{c}87,0 \\
(85,0 \text { à } 88,9)\end{array}$ & $\begin{array}{c}29,7 \\
(26,7 \text { à } 32,7)\end{array}$ & $\begin{array}{c}70,3 \\
(67,3 \text { à } 73,3)\end{array}$ \\
\hline Vivant en couple $^{\mathrm{a}}$ & $\begin{array}{c}51,6 \\
(49,2 \text { à } 54,0)\end{array}$ & $\begin{array}{c}48,4 \\
(46,0 \text { à } 50,8)\end{array}$ & $\begin{array}{c}25,0 \\
(22,8 \text { à } 27,2)\end{array}$ & $\begin{array}{c}75,0 \\
(72,8 \text { à } 77,2)\end{array}$ & $\begin{array}{c}6,2 \\
(4,9 \text { à } 7,4)\end{array}$ & $\begin{array}{c}93,8 \\
(92,6 \text { à } 95,1)\end{array}$ & $\begin{array}{c}22,0 \\
(19,9 \text { à } 24,1)\end{array}$ & $\begin{array}{c}78,0 \\
(75,9 \text { à } 80,1)\end{array}$ \\
\hline Autres $^{\mathrm{b}}$ & $\begin{array}{c}61,1 \\
(53,1 \text { à } 69,1)\end{array}$ & $\begin{array}{c}38,9 \\
(30,9 \text { à } 46,9)\end{array}$ & $\begin{array}{c}30,2 \\
(22,3 \text { à } 38,1)\end{array}$ & $\begin{array}{c}69,8 \\
(61,9 \text { à } 77,7)\end{array}$ & $\begin{array}{c}8,7 \\
(4,9 \text { à } 12,5)\end{array}$ & $\begin{array}{c}91,3 \\
(87,5 \text { à } 95,1)\end{array}$ & $\begin{array}{c}27,6 \\
(19,1 \text { à } 36,1)\end{array}$ & $\begin{array}{c}72,4 \\
(63,9 \text { à } 80,9)\end{array}$ \\
\hline
\end{tabular}

Abréviations : ESCC, Enquête sur la santé dans les collectivités canadiennes; IC, intervalle de confiance.

Remarques : Sauf indication contraire, les données sont exprimées en pourcentage pour la rangée (IC à $95 \%$ ).

a Vivant avec ou sans enfants.

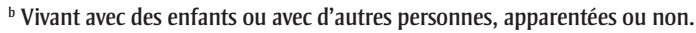

faible. Comparativement à celles qui vivaient en couple, les femmes vivant seules et celles vivant avec des enfants ou d'autres personnes (apparentées ou non) ont fait état d'une moins bonne santé générale et d'une moins bonne santé mentale. De plus, les femmes vivant seules se sont déclarées moins satisfaites de leur vie, tandis que celles qui vivaient avec des enfants ou d'autres personnes (apparentées ou non) avaient un sentiment plus faible d'appartenance à la collectivité. Chez les hommes, ceux qui vivaient seuls ont déclaré avoir une santé mentale, une satisfaction envers la vie et un sentiment d'appartenance à la collectivité moins bons que ceux qui vivaient en couple. L'état de santé général perçu ne différait pas entre ces trois conditions de logement pour les hommes. Les hommes vivant avec des enfants ou avec d'autres personnes (apparentées ou non) n’étaient pas plus susceptibles de déclarer une moins bonne santé et un bien-être social plus faible que ceux vivant en couple, et ce, pour les quatre mesures.

\section{Analyse}

D’après les données de l'ESCC de 2018, près de la moitié des aînés vivent en couple (49,2\% au total). D’autres études ont révélé que la cohabitation avec d'autres personnes peut être particulièrement bénéfique pour vieillir en santé et que le fait de vivre seul peut être préjudiciable ${ }^{7-10}$. Le nombre de personnes vivant seules semble néanmoins être à la hausse dans ce groupe d'âge : dans une analyse du recensement de 2011, on a constaté que $31,5 \%$ des femmes et $16 \%$ des hommes de 65 ans et plus vivaient seuls ${ }^{11}$, ces chiffres étant passé à $33 \%$ et à $17,4 \%$ dans le recensement de $2016^{12}$, puis à 35,7\% et à 19,1\% dans notre analyse de l'ESCC de 2018.

Parmi les aînés, les femmes, les personnes plus âgées, à faible revenu, divorcées ou séparées, vivant en milieu rubain, locataires et moins scolarisées étaient plus susceptibles de vivre seules. Des résultats similaires ont été signalés ailleurs. On remarque régulièrement que les aînées sont plus susceptibles de vivre seules, tant au Canada ${ }^{11,12}$ que dans d'autres pays ${ }^{16,17}$, en partie en raison de leur espérance de vie plus longue que celle des conjoints de sexe opposé ${ }^{11}$. Selon d'autres études, les aînés à faible revenu ${ }^{17,18}$ ou peu scolarisés ${ }^{16}$ sont plus susceptibles de vivre seuls, ce qui pourrait être attribuable à leur incapacité à assumer le coût élevé d'un logement avec services ${ }^{19}$. En outre, les aînés vivant en milieu urbain sont plus susceptibles de vivre seuls, peut-être en raison de la disparité des services et des offres de soutien proposés aux aînés en milieu rural $^{20}$. En fait, les services pour aînés les plus utilisés sont les centres pour personnes âgées, les services d'aide-ménagère et les services de transport ${ }^{21}$, qui sont tous plus susceptibles d'être offerts en milieu urbain. Enfin, nous avons pu constater, à la suite d'autres études, que le fait de vivre seul était associé à une plus mauvaise perception de la santé et du bienêtre social chez les aînés. Cela dit, la temporalité de cette association et des autres associations mentionnées ne peut pas être déterminée au moyen de cette analyse transversale. Il demeure que le fait de cibler cette sous-population d'aînés vulnérables va nous aider à élaborer des politiques et des programmes plus efficaces pour promouvoir le vieillissement en santé. Bien sûr, d'autres facteurs comme le désir ou le choix de vivre seul et le capital social devraient être pris en considération pour cibler plus spécifiquement les personnes les plus à risque. 


\section{Points forts et limites}

Notre analyse est fondée sur les données transversales d'un questionnaire, ce qui rend les inférences causales difficiles. De plus, les données sur les aînés résidant dans les territoires ou en établissement n'ont pas été incluses, ce qui signifie qu'on ne peut présumer des conditions de logement des aînés vivant dans ces régions ou dans ces circonstances. Il demeure que nous avons disposé d'un échantillon de taille importante et qui a été pondéré pour être représentatif de la population canadienne des provinces et de celle ne résidant pas en établissement. De plus, notre analyse utilise les données les plus récentes sur les aînés canadiens.

\section{Conclusion}

Notre analyse a révélé que, parmi les aînés, les femmes, les personnes plus âgées, à faible revenu, divorcées ou séparées, vivant en milieu urbain, locataires, moins scolarisées et ayant une mauvaise perception de leur santé et de leur bien-être social étaient plus susceptibles de vivre seules et étaient potentiellement plus vulnérables aux problèmes de santé en vieillissant. Ce constat pourrait aider les programmes et les politiques à cibler les aînés qui courent un risque plus élevé de souffrir de problèmes de santé en raison de leurs conditions de logement, et ainsi d'accroître la prévalence du vieillissement en santé chez les aînés canadiens. Par exemple, les responsables des politiques en matière de santé pourraient promouvoir la mise sur pied de programmes communautaires visant à accroître la participation et l'inclusion sociales des femmes âgées vivant seules afin d'accroitre leur sentiment d'appartenance à la collectivité.

\section{Remerciements}

Nous aimerions remercier Kerry Anderson, Patti Gorr et Rachel Milliken de la Division du vieillissement et des aînés du Centre pour la promotion de la santé de l'ASPC pour leur contribution. Leur compréhension approfondie des enjeux auxquels font face les aînés canadiens a éclairé l'analyse et l'interprétation des résultats. Nous aimerions également remercier le $\mathrm{D}^{\mathrm{r}}$ Howard Morrison pour ses conseils professionnels.

\section{Conflits d'intérêts}

Les auteurs déclarent n'avoir aucun conflit d'intérêts. Cette recherche n'a reçu aucun financement.

\section{Contribution des auteurs et avis}

Tous les auteurs ont conçu la méthodologie. SAS a effectué les analyses et rédigé la version préliminaire du document. Tous les auteurs ont interprété les résultats et procédé à une révision critique de l'article. Tous les auteurs ont lu et approuvé la version définitive.

Les auteurs assument la responsabilité du contenu de l'article et des points de vue qui y sont exprimés; ceux-ci ne reflètent pas nécessairement ceux du gouvernement du Canada.

\section{Références}

1. Shumanty R. Rapport sur l'état de la population du Canada. Mortalité : aperçu, 2014 à 2016. Ottawa (Ont.) : Statistique Canada; 2018. 14 p. [n ${ }^{\circ}$ de catalogue : 91-209-X]

2. Provencher C, Milan A, Hallman S, et al. Rapport sur l'état de la population du Canada. Fécondité : aperçu, 2012 à 2016. Ottawa (Ont.) : Statistique Canada; 2018. 19 p. [n ${ }^{\circ}$ de catalogue : 91-209-X]

3. Statistique Canada. Estimations démographiques annuelles : Canada, provinces et territoires, 2018. Analyse : Population selon l'âge et le sexe [Internet]. Ottawa (Ont.) : Statistique Canada; 2018 [consultation le 29 mai 2019]. En ligne à : https://www150 .statcan.gc.ca/n1/pub/91-215-x/2018002 /sec2-fra.htm

4. Statistique Canada. Le Quotidien Estimations de la population du Canada : âge et sexe, $1^{\text {er }}$ juillet 2018 [Internet]. Ottawa (Ont.) : Statistique Canada; 25 janvier 2019 [consultation le 29 mai 2019]. En ligne à : https://www 150 .statcan.gc.ca/n1/daily-quotidien/190125 /dq190125a-fra.htm

5. Meng X, D’Arcy C. Successful aging in Canada: prevalence and predictors from a population-based sample of older adults. Gerontology. 2014;60(1): 65-72.

6. Rowe JW, Kahn RL. Successful aging. Gerontologist. 1997;37(4):433-440.

7. Arai A, Katsumata Y, Konno K, et all. Sociodemographic factors associated with incidence of dementia among senior citizens of a small town in Japan. Care Manag J. 2004;5(3):159-165.
8. Müller F, Hagedoorn M, Tuinman MA. Chronic multimorbidity impairs role functioning in middle-aged and older individuals mostly when nonpartnered or living alone. PLOS ONE [Internet]. 2017 [consultation le 29 mai 2019]; 12(2):e0170525. En ligne à : https://journals.plos.org/plosone/article ? id $=10.1371 /$ journal.pone. 0170525

9. Agrawal S. Effect of living arrangement on the health status of elderly in India: findings from a national cross sectional survey. Asian Popul Stud. 2012;8(1):87-101

10. Yu C-Y, Hou S-I, Miller J. Health for older adults: the role of social capital and leisure-time physical activity by living arrangements. J Phys Act Health. 2018;15(2):150-158

11. Statistique Canada. La situation des personnes âgées dans les ménages [Internet]. Ottawa (Ont.) : Gouvernement du Canada; 2012 [consultation le 21 mai 2019]. En ligne à : https://www12.statcan.gc.ca/census -recensement/2011/as-sa/98-312-x /98-312-x2011003_4-fra.cfm

12. Tang J, Galbraith N, Truong J. Vivre seul au Canada. Ottawa (Ont.) : Statistique Canada; 2019. [n ${ }^{\circ}$ de catalogue : 75-006-X]

13. Gee EM. Living arrangements and quality of life among Chinese Canadian elders. Soc Indic Res. 2000;51(3):309-329.

14. Ng CF, Northcott HC, Abu-Laban SM. Housing and living arrangements of South Asian Immigrant Seniors in Edmonton, Alberta. Can J Aging. 2007; 26(3):185-194.

15. Statistique Canada. Enquête sur la santé dans les collectivités canadiennes (ESCC) - Composante annuelle. Guide de l'utilisateur. Fichier de microdonnées de 2018. Ottawa (Ont.) : Gouvernement du Canada; 2018.

16. You KS, Lee H. The physical, mental, and emotional health of older people who are living alone or with relatives. Arch Psychiatr Nurs. 2006;20(4):193-201.

17. Beckett MK, Elliott MN, Haviland AM, Burkhart Q, Gaillot S, Montfort $\mathrm{D}$, et al. Living alone and patient care experiences: the role of gender in a national sample of Medicare beneficiaries. J Gerontol A. 2015;70(10): 1242-1247. 
18. Administration on Aging. A profile of older Americans: 2011. Washington (DC): US Department of Health and Human Services; 2011. En ligne à : https://acl.gov/sites/default/files /Aging \% 20and \% 20Disability \% 20 in \%20America/2011profile.pdf

19. White G, Singh T, Caine K, Connelly K. Limited but satisfied: low SES older adults' experiences of aging in place. Dans : Proceedings of the 9th International Conference on Pervasive Computing Technologies for Healthcare. 20 au 23 mai 2015; Istanbul, Turquie : 121-128.

20. Siconolfi D, Shih RA, Friedman EM, et al. Rural-urban disparities in access to home- and community-based services and supports: stakeholder perspectives from 14 states. J Am Med Dir Assoc. 2019;20(4):503-508.e1.

21. Ewen HH, Washington TR, Emerson $\mathrm{KG}$, et al. Variation in older adult characteristics by residence type and use of home- and community-based services. Int $\mathrm{J}$ Environ Res Public Health [Internet]. 2017 [consultation le 28 mai 2019]; 14(3):330. En ligne à : https://www.mdpi.com/1660-4601 $/ 14 / 3 / 330$ 